\section{POTENCIAL DE RENDIMIENTO DE POBLACIONES CRIOLLAS DE MAÍZ DE COAHUILA, MÉXICO}

\section{YIELD POTENTIAL OF MAIZE LANDRACES FROM COAHUILA, MÉXICO}

\author{
Luis A. Nájera Calvo ${ }^{1}$, Froylán Rincón Sánchez ${ }^{1^{*}}$, \\ Norma A. Ruiz Torres ${ }^{2}$ y Fernando Castillo \\ González $^{3}$
}

\begin{abstract}
${ }^{1}$ Departamento de Fitomejoramiento y ${ }^{2}$ Centro de Capacitación y Desarrollo de Tecnología de Semillas (CCDTS), Universidad Autónoma Agraria Antonio Narro. Calzada Antonio Narro 1923. 25315, Buenavista, Saltillo, Coahuila. Tel. (844) 4110220. ${ }^{3}$ Postgrado en Recursos Genéticos y Productividad, Colegio de Postgraduados. Km 36.5 Carr. México-Texcoco. 56230, Montecillo, Texcoco, Estado de México.
\end{abstract}

* Autor para correspondencia (frincon@uaaan.mx)

\section{RESUMEN}

Las poblaciones y tipos de maíces (Zea mays L.) adaptados a condiciones de temporal o secano poseen atributos que pueden ser útiles en el mejoramiento genético. En el Estado de Coahuila, México, se ha identificado la presencia de siete grupos raciales. El objetivo del presente trabajo fue evaluar agronómicamente y determinar el potencial de rendimiento de grano de 90 poblaciones de maíces criollos recolectadas en el Estado de Coahuila. La evaluación agronómica se hizo en 2008 y 2009, en dos localidades: El Mezquite, Galeana, Nuevo León $(1890 \mathrm{~m})$ y General Cepeda, Coahuila $(1350 \mathrm{~m})$. La combinación de años y localidades fue considerada como cuatro ambientes (MEZ08, MEZ09, GC08 y GC09). Las poblaciones fueron agrupadas de acuerdo con la altitud de procedencia en: bajas $(0$ a $1000 \mathrm{~m})$, intermedias $(1001$ a $1800 \mathrm{~m})$, transición $(1801$ a $2000 \mathrm{~m})$ y de altura (más de $2000 \mathrm{~m})$. Los resultados mostraron diferencias $(P \leq 0.01)$ entre grupos y en la interacción grupos $x$ ambientes, para floración masculina $y$ rendimiento de grano; también se encontraron diferencias $(P \leq 0.01)$ entre poblaciones dentro de grupos y en poblaciones dentro de grupo $\mathrm{x}$ ambientes. El análisis de la interacción poblaciones $\mathrm{x}$ ambientes permitió identificar tres grupos según su adaptación a las localidades: el primero, con adaptación a El Mezquite (33.3 \%), el segundo a General Cepeda (42.2 \%), y el tercer grupo (24.4\%) con estabilidad a través de ambientes. Las poblaciones 19, 22, 34 y 61 tuvieron adaptación a El Mezquite; las poblaciones 74,76 y 88 a General Cepeda; y las poblaciones 35, 37, 38, 64, 66 y 81 mostraron estabilidad a través de ambientes. Los grupos raciales con mayor potencial de rendimiento fueron Tuxpeño, Tuxpeño Norteño $y$ Ratón.
Palabras clave: Zea mays, evaluación de germoplasma, diversidad genética, poblaciones criollas.

\section{SUMMARY}

Types and populations of maize (Zea mays L.) adapted to rain fed conditions might have attributes useful for plant breeding. Seven racial groups have been identified in the State of Coahuila, México. The objective of this research was to evaluate agronomic traits and grain yield potential of 90 maize populations collected in the State of Coahuila. The evaluation was performed at two locations: El Mezquite, Galeana, Nuevo León $(1890 \mathrm{~m})$ and General Cepeda, Coahuila $(1350 \mathrm{~m})$. The locations $x$ years combination was considered as four environments (MEZ08, MEZ09, GC08, GC09). Populations were grouped according to provenance altitude: low (0 to $1000 \mathrm{~m})$, intermediate $(1001$ to $1800 \mathrm{~m})$, transition $(1801$ a 2000 $\mathrm{m})$ and high (higher than $2000 \mathrm{~m}$ ). Results showed differences $(\mathrm{P} \leq$ 0.01) among groups and among groups $x$ Environments interaction, for male flowering and grain yield; differences $(P \leq 0.01)$ between populations were also found within groups and populations within group $x$ environments. The populations $x$ environments interaction analysis allowed the identification of three groups according to adaptation to localities: the first one was adapted to El Mezquite $(33.3 \%)$, the second one to General Cepeda (42.2 \%), and the third group (24.4 \%) had stability across environments. Populations 19, 22, 34 and 61 were adapted to El Mezquite; populations 74, 76 and 88 to General Cepeda; and populations 35, 37, 38, 64, 66 and 81 had stability across environments. The racial groups with highest yield potential were Tuxpeño, Tuxpeño Norteño and Raton.

Index words: Zea mays, germplasm evaluation, genetic diversity, landrace populations.

\section{INTRODUCCIÓN}

En México, el maíz (Zea mays L.) es el cultivo agrícola más importante desde el punto de vista alimentario, industrial y social (SAGARPA-SIAP, 2007), y en el país se encuentra la mayor riqueza genética documentada en los diferentes complejos raciales (Wellhausen et al., 1951). La variación genética está estrechamente relacionada con las condiciones ambientales de producción, así como con la diversidad de usos que se da al maíz, especialmente al grano (Hernández y Esquivel, 2004).

El conocimiento de la variación morfológica y genética, su relación con el ambiente y el impacto social determinan la estrategia de conservación y aprovechamiento de la diversidad genética del maíz (Ortega et al., 1991). La variación genética permite integrar poblaciones de amplia base genética, las cuales sirven como material base en los programas de mejoramiento genético para incrementar el rendimiento y caracteres agronómicos de interés si son incorporados a nuevas variedades mejoradas sintéticos o híbridos (García et al., 2002). Sin embargo, el uso real de la variación genética es muy limitado, pues de las 50 razas de maíz reportadas solamente se utilizan cuatro en mejoramiento genético: Chalqueño en la Mesa Central, Tuxpeño en el Trópico seco y húmedo, Cónico para zonas 
con problemas pluviales de la Mesa Central, y Celaya para El Bajío y zonas similares (Márquez, 2005).

La preocupación por la pérdida de la diversidad ha dado lugar a investigaciones destinadas a describir y comprender los factores que influyen en la diversidad de las poblaciones de maíz de los agricultores mexicanos (Aguirre et al., 1998).

La recolección y estudio de los maíces criollos mexicanos han sido motivados por diversas causas: contar con fuentes de germoplasma para mejoramiento genético, entender la agricultura mexicana de autoconsumo, realizar estudios biológicos básicos para entender el proceso evolutivo, realizar evaluaciones preliminares, y aplicar técnicas de premejoramiento (Hernández y Esquivel, 2004). Históricamente se han hecho colectas de maíces nativos en la mayor parte de las regiones importantes de México (Wellhausen et al., 1951). Adicionalmente, la información disponible en los bancos de germoplasma del Instituto Nacional de Investigaciones Forestales, Agrícolas y Pecuarias (INIFAP) y del Centro Internacional de Mejoramiento de Maíz y Trigo
(CIMMYT), más otros trabajos adicionales, documentan la presencia de por lo menos cuatro grupos raciales en Coahuila: Cónico Norteño, Tuxpeño, Ratón y Tuxpeño Norteño. El objetivo del presente trabajo fue evaluar agronómicamente y determinar el potencial de rendimiento de 90 poblaciones de maíces criollos recolectadas en Coahuila.

\section{MATERIALES Y MÉTODOS}

El material genético utilizado en el estudio proviene de la colecta de 90 poblaciones de maíces realizada en 2008 en 23 municipios productores de maíz en el Estado de Coahuila, México. Una muestra de mazorcas de estos materiales se presenta en la Figura 1. La clasificación preliminar identificó a siete grupos raciales: Celaya, Cónico Norteño, Elotes Cónicos, Olotillo, Ratón, Tuxpeño y Tuxpeño Norteño, con 1, 22, 2, 3, 33, 9 y 20 poblaciones, respectivamente. Las poblaciones fueron colectadas en altitudes de 248 a $2557 \mathrm{~m}$, y agrupadas de acuerdo con la altitud de procedencia en: bajas ( 0 a 1000 $\mathrm{m})$, intermedias (1001 a $1800 \mathrm{~m})$, transición (1801 a 2000 m) y de altura (más de 2000 m) (Cuadro 1).

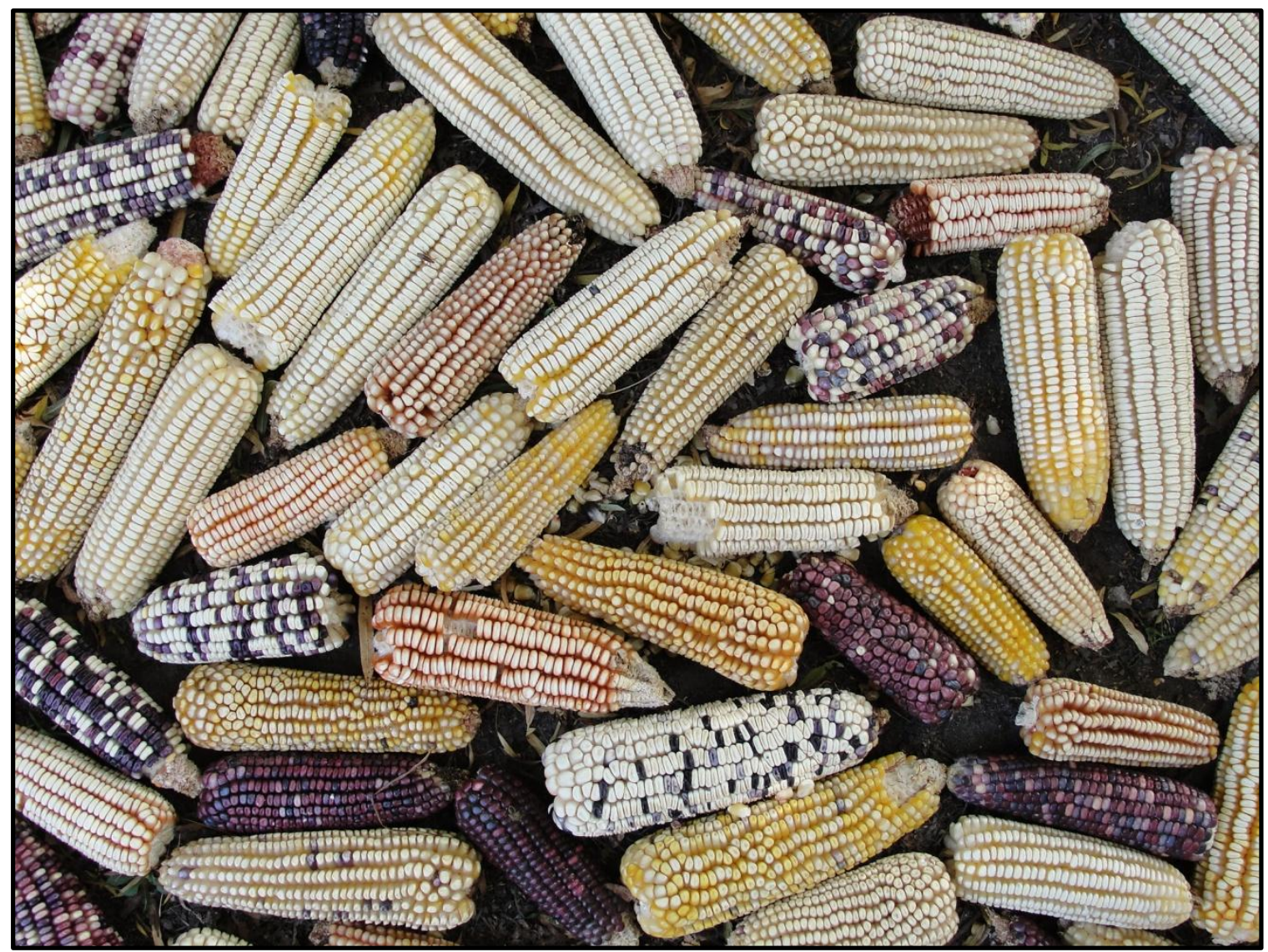

Figura 1. Muestra de mazorcas de maíces criollos del Estado de Coahuila, México. 
La evaluación agronómica se llevó a cabo en 2008 y 2009 en dos localidades: El Mezquite, Galeana, N. L. (1890 m; 25 05' LN; $100^{\circ} 42^{\prime}$ LO) y General Cepeda, Coah. (1350 m; 25 26' LN; 101 ${ }^{\circ} 27^{\prime}$ LO), en condiciones de riego. La combinación de años y localidades fue considerada como cuatro ambientes de evaluación (MZ08, MZ09, GC08 y GC09, respectivamente). Las poblaciones se establecieron en experimentos repetidos en surcos de $4 \mathrm{~m}$ de largo, con una distancia entre plantas de $0.2 \mathrm{~m}$ y distancia entre surcos de $0.92 \mathrm{~m}$ en El Mezquite, y de $0.80 \mathrm{~m}$ en General Cepeda, en los dos años. Se utilizó un diseño de bloques incompletos con arreglo $\alpha$-látice (Barreto et al., 1997), con dos repeticiones dentro de localidades $\mathrm{x}$ años. Los experimentos fueron generados con el paquete CropStat (IRRI, 2007).

Las variables analizadas en el presente documento fueron: días a floración masculina (FM), altura de planta (AP) y rendimiento de grano (REND, en $\mathrm{t} \mathrm{ha}^{-1} \mathrm{y}$ ajustado a $15 \%$ de humedad). Se hizo un análisis de varianza mediante el paquete SAS (SAS Institute, 2004), donde se probaron los efectos de grupos, poblaciones dentro de grupos, y las interacciones con los ambientes de evaluación. En el análisis de varianza, los ambientes, grupos $\mathrm{y}$ la interacción grupos $\mathrm{x}$ ambientes fueron considerados efectos fijos, y el resto como efectos aleatorios. En la comparación de medias, se utilizó como criterio de selección de poblaciones al valor superior a la $\mu+2$ el error estándar. Los datos de repeticiones, localidades y años (MEZ08, MEZ09, GC08 y GC09) se usaron para el análisis de la interacción poblaciones $\mathrm{x}$ ambientes, mediante un análisis de dispersión gráfica (Yan y Tinker, 2006; Yan, 2007).

\section{RESULTADOS Y DISCUSIÓN}

Se detectaron diferencias $(\mathrm{P} \leq 0.01)$ entre grupos y efecto de la interacción grupos $\mathrm{x}$ ambientes para floración masculina y rendimiento de grano. También se encontró diferencia $(\mathrm{P} \leq 0.01)$ entre poblaciones dentro de grupos y entre poblaciones dentro de grupo $\mathrm{x}$ ambientes (Cuadro 2). Lo anterior indica la existencia de diversidad genética entre poblaciones adaptadas a áreas ecológicas específicas (Cuadro 1).

Las principales razas representadas fueron Ratón, Cónico Norteño y Tuxpeño Norteño, las cuales cuentan con una distribución amplia; el resto de las razas constituye un componente importante en cada una de las regiones ecológicas. Aun cuando se encontró una distribución amplia de los grupos raciales, el comportamiento agronómico de las poblaciones mostró un patrón asociado con el origen y condiciones de adaptación.

Cuadro 1. Clasificación de razas de maíz en grupos, y número de poblaciones por grupo, con base en la altitud del sitio de colecta, Coahuila, México, 2008.

\begin{tabular}{lccrr}
\hline \multirow{2}{*}{ Raza } & \multicolumn{5}{c}{ Grupos (por altitud, en m) } \\
\cline { 2 - 5 } & $\begin{array}{c}\text { Baja (B) } \\
(<1000)\end{array}$ & $\begin{array}{c}\text { Intermedia (I) } \\
(1001-1800)\end{array}$ & $\begin{array}{c}\text { De transición (T) } \\
(1801-2000)\end{array}$ & $\begin{array}{c}\text { De altura (A) } \\
(>2001)\end{array}$ \\
\hline Celaya & 1 & & & $\mathbf{1}$ \\
Cónico Norteño & & 2 & 7 & 13 \\
Elotes Cónicos & & & 1 & 1 \\
Olotillo & 9 & 3 & 1 & $\mathbf{2 2}$ \\
Ratón & 7 & 23 & & $\mathbf{3}$ \\
Tuxpeño & 11 & 2 & 1 & $\mathbf{3 3}$ \\
Tuxpeño Norteño & $\mathbf{2 8}$ & $\mathbf{3 8}$ & $\mathbf{1 0}$ & $\mathbf{2 0}$ \\
Total & & & $\mathbf{9 0}$ \\
\hline
\end{tabular}

Cuadro 2. Cuadrados medios del análisis de varianza de las 90 poblaciones evaluadas en cuatro ambientes en 2008 y 2009.

\begin{tabular}{lcccc}
\hline Fuente de variación & $\mathrm{gl}$ & $\begin{array}{c}\text { Rendimiento de grano } \\
(\mathrm{t} \mathrm{ha})\end{array}$ & $\begin{array}{c}\text { Floración masculina } \\
(\mathrm{d})\end{array}$ & $\begin{array}{c}\text { Altura de planta } \\
(\mathrm{cm})\end{array}$ \\
\hline Ambientes (Amb) & 3 & $195.2^{* *}$ & $26217.6^{* *}$ & $25942.6^{* *}$ \\
$\quad$ Repeticiones (Rep) /Amb & 4 & 3.2 & 57.9 & 1052.8 \\
$\quad$ Bloques/(Rep x Amb) & 52 & $1.8^{*}$ & $29.7 * *$ & $682.0 * *$ \\
Grupos (Gpo) & 3 & $126.6^{* *}$ & $4241.9 * *$ & $12962.0 *$ \\
$\quad$ Poblaciones (Pob)/Gpo & 86 & $6.4^{* *}$ & $217.2 * *$ & $4242.2 * *$ \\
Gpo x Amb & 9 & $33.6 * *$ & $263.4 * *$ & $842.2 * *$ \\
Pob / (Gpo x Amb) & 258 & $1.8 * *$ & $19.8^{* *}$ & $320.0 * *$ \\
Error & 304 & 1.2 & 13.5 & 245.1 \\
& $\mathrm{CV}(\%)$ & & 4.2 & 6.8 \\
\hline
\end{tabular}

*, **, Significativo al 0.05 y 0.01 niveles de probabilidad, respectivamente; gl $=$ grados de libertad. 
La raza Cónico Norteño cuenta con adaptación entre el área de transición y de altura, en tanto que las razas Ratón y Tuxpeño Norteño se adaptan a las áreas de bajas a intermedias, principalmente (Cuadro 1). Según Castillo (1993), la variabilidad genética se puede estudiar para determinar la dirección de su aprovechamiento, para lograr un mejor uso de los genotipos y relacionarlos con su área de adaptación.

Las poblaciones evaluadas tuvieron un comportamiento diferente $(\mathrm{P} \leq 0.01)$ en los ambientes de evaluación, lo cual era de esperarse por la diversidad de sitios de procedencia y de las combinaciones genéticas presentes en ellas. La respuesta de interacción poblaciones $\mathrm{x}$ ambientes (Figura 2), puede ser útil para identificar a las poblaciones con adaptación específica a las condiciones de los sitios incluidos en el estudio. Dicha figura representa la dispersión de las poblaciones con respecto a los años de evaluación y localidades (MZ08, MZ09, GC08 y GC09, respectivamente), con una explicación de $86.1 \%$ de la variación total en el rendimiento de grano. En el eje de las abscisas se indica el rendimiento promedio de las poblaciones, en tanto que el eje de las ordenadas corresponde a la respuesta específica de las poblaciones en los ambientes de evaluación (Yan y Tinker, 2006). Como los ambientes de evaluación son los probadores de las poblaciones, el eje inclinado de la abscisa indica los efectos de aptitud combinatoria general (ACG) y de la ordenada a la aptitud combinatoria específica (ACE), según Yan y Kang (2003).

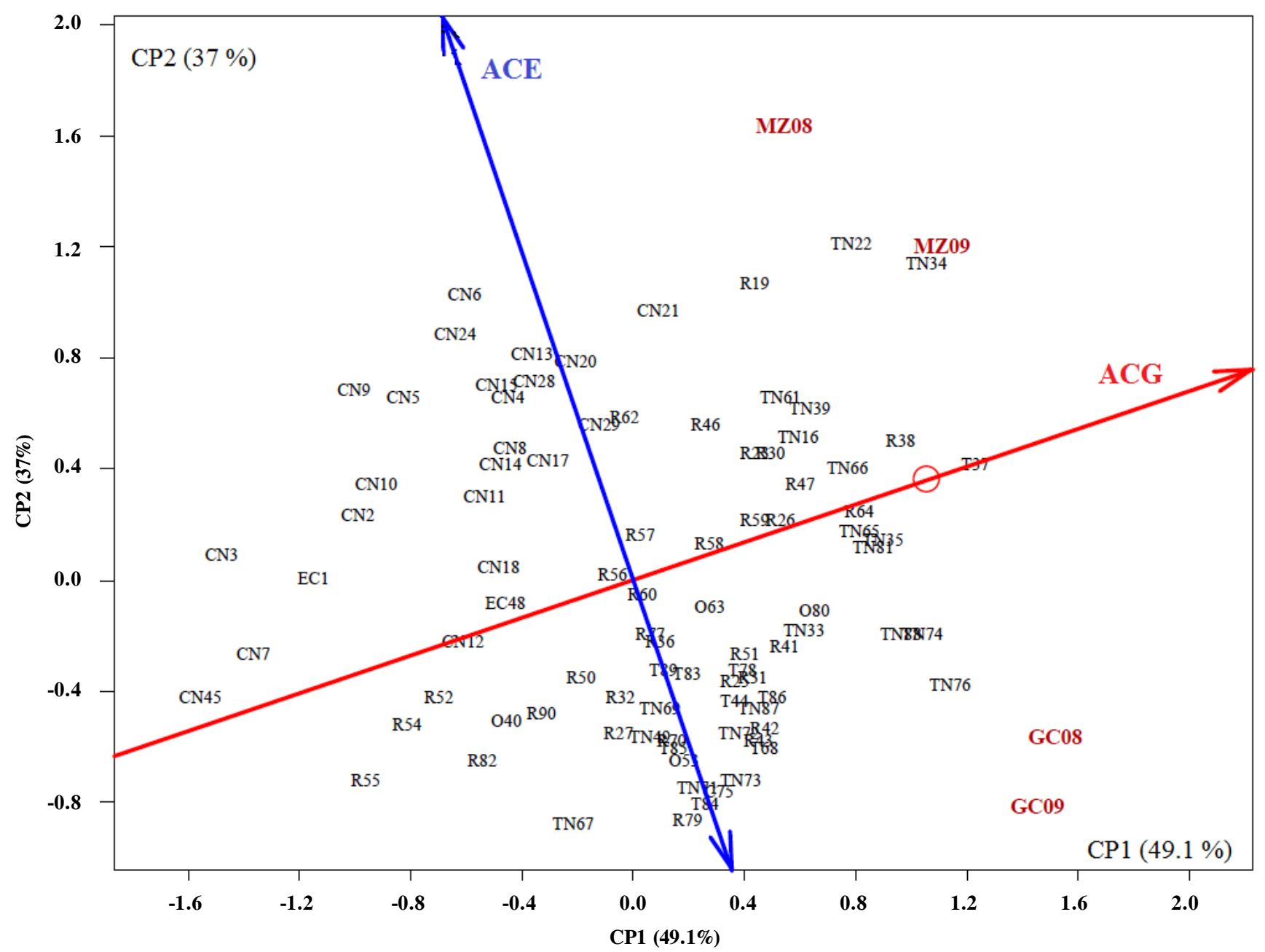

Figura 2. Interacción poblaciones $\mathrm{x}$ ambientes de evaluación, del rendimiento de grano de 90 poblaciones de maíz evaluadas en dos localidades de Coahuila, México, durante 2008 y 2009 (MZ08, MZ09, GC08 y GC09). Los puntos representan al grupo racial (letra) y a la población (número). $\mathrm{C}=$ Celaya; $\mathrm{CN}=$ Cónico Norteño; $\mathbf{E C}=$ Elotes Cónicos; $\mathbf{O}=$ Olotillo; $\mathbf{R}=\mathbf{R a t o ́ n} ; \mathbf{T}=\mathbf{T u x p e n ̃ o ; ~ T N ~ = ~ T u x p e n ̃ o ~}$ Norteño. ACG = Aptitud combinatoria general; ACE = Aptitud combinatoria específica. 
El ángulo que forman los vectores de los ambientes a partir del origen, indica el nivel de asociación entre ellos. Por tanto, los vectores asociados a cada ambiente (localidad $\mathrm{x}$ año) están estrechamente relacionados (ángulo menor a $30^{\circ}$ ), en tanto que entre las dos localidades, los ambientes muestran un comportamiento independiente entre ellos (ángulo de aproximadamente $\left.90^{\circ}\right)$. La ubicación de las poblaciones en las ordenadas con dirección alejada del origen, indican la respuesta positiva al ambiente específico; por tanto, las poblaciones ubicadas cerca de la línea paralela a las abscisas, son consideradas como estables en rendimiento de grano a través de ambientes (localidades $\mathrm{x}$ años) (Yan y Tinker, 2006).

Cuadro 3. Medias de rendimiento de grano y floración masculina de las poblaciones de maíz con mayor rendimiento a través de ambientes de evaluación, en 2008 y 2009.

\begin{tabular}{|c|c|c|c|c|c|c|c|c|c|c|c|}
\hline \multirow[t]{2}{*}{ Población } & \multirow[t]{2}{*}{ Grupo } & \multirow[t]{2}{*}{ Raza } & \multicolumn{5}{|c|}{ Rendimiento de grano $\left(\mathrm{t} \mathrm{ha}^{-1}\right)$} & \multicolumn{4}{|c|}{ Floración masculina (d) } \\
\hline & & & Promedio & GC08 & GC09 & MZ08 & MZ09 & GC08 & GC09 & MZ08 & MZ09 \\
\hline$\overline{\mathrm{COAH}-037}$ & I & $\mathrm{T} \times \mathrm{C}$ & $8.02 *$ & $6.22 *$ & $8.26 *$ & 6.22 & $11.37 *$ & 81.0 & $77.5 *$ & $106.0 *$ & 107.0 \\
\hline $\mathrm{COAH}-076$ & B & $\mathrm{TN} \times \mathrm{C}$ & $7.82 *$ & $7.07 *$ & $9.72 *$ & 6.08 & 8.40 & $85.5 *$ & $80.5 *$ & $111.5 *$ & $116.0 *$ \\
\hline COAH-034 & I & $\mathrm{TN}$ & $7.82 *$ & 5.63 & 6.87 & $8.67 *$ & $10.09 *$ & 78.0 & 70.5 & 94.0 & 101.0 \\
\hline COAH-038 & B & $\mathrm{R} \times \mathrm{TN}$ & $7.77 *$ & $6.27 *$ & $7.22 *$ & $8.03 *$ & $9.56 *$ & $85.5 *$ & 73.0 & $105.0 *$ & 105.0 \\
\hline COAH-081 & B & $\mathrm{TN}$ & $7.65 *$ & 5.63 & $8.21 *$ & 6.01 & $10.74 *$ & $89.0 *$ & $84.5 *$ & $111.5 *$ & $121.0 *$ \\
\hline COAH-074 & B & $\mathrm{TN} \times \mathrm{R}$ & $7.54 *$ & 5.65 & $9.48 *$ & 5.62 & $9.42 *$ & 78.0 & $77.0 *$ & $108.5 *$ & $111.5 *$ \\
\hline COAH-088 & B & $\mathrm{TN}$ & $7.50 *$ & $8.88 *$ & 6.64 & 5.78 & 8.72 & 80.0 & 73.0 & $108.0 *$ & $108.5 *$ \\
\hline $\mathrm{COAH}-022$ & I & $\mathrm{TN}$ & $7.47 *$ & $6.74 *$ & 4.13 & $8.90 *$ & $10.13 *$ & 79.0 & $75.0 *$ & 98.0 & 99.0 \\
\hline COAH-035 & I & $\mathrm{TN}$ & $7.40 *$ & $6.74 *$ & $7.42 *$ & $7.34 *$ & 8.10 & $83.0 *$ & 72.0 & 103.5 & $111.0 *$ \\
\hline COAH-065 & I & $\mathrm{TN}$ & $7.34 *$ & 5.81 & $7.89 *$ & 6.97 & 8.68 & $83.0 *$ & $79.0 *$ & 104.0 & $110.5 *$ \\
\hline COAH-066 & I & $\mathrm{TN}$ & $7.29 *$ & $7.22 *$ & 5.71 & 7.05 & $9.16 *$ & 80.0 & 73.0 & 103.5 & 103.0 \\
\hline COAH-064 & I & $\mathrm{R} \times \mathrm{TN}$ & $7.23 *$ & $6.24 *$ & 6.99 & 6.20 & $9.50 *$ & $82.5 *$ & 73.5 & 101.5 & 102.0 \\
\hline СOAH-039 & B & $\mathrm{TN}$ & $7.13 *$ & $7.40 *$ & 4.93 & $8.68 *$ & 7.50 & $81.5 *$ & $79.0 *$ & 100.0 & $111.0 *$ \\
\hline COAH-061 & I & $\mathrm{TN}$ & $6.96 *$ & 5.26 & 5.76 & $7.77 *$ & $9.04 *$ & 76.5 & 72.0 & 92.0 & 101.0 \\
\hline COAH-019 & $\mathrm{T}$ & $\mathrm{R}$ & $6.89 *$ & 6.09 & 4.32 & $9.57 *$ & 7.60 & 81.0 & $77.0 *$ & 94.0 & 98.0 \\
\hline COAH-086 & B & $\mathrm{T}$ & $6.85 *$ & 5.88 & $8.28 *$ & 5.46 & 7.78 & $90.0 *$ & $87.5 *$ & 118.0 * & $128.0 *$ \\
\hline COAH-080 & B & $\mathrm{R} \times \mathrm{O}$ & $6.83 *$ & 5.70 & $7.69 *$ & 5.75 & 8.21 & 79.0 & 69.0 & 98.5 & 106.0 \\
\hline COAH-016 & $\mathrm{T}$ & $\mathrm{TN}$ & $6.81 *$ & 5.74 & 5.73 & $7.63 *$ & 8.13 & 79.0 & 69.0 & 100.0 & 94.5 \\
\hline COAH-047 & I & $\mathrm{R}$ & $6.69 *$ & 5.39 & 5.95 & 6.15 & $9.28 *$ & 78.0 & 71.5 & 98.0 & 101.0 \\
\hline COAH-033 & B & $\mathrm{TN}$ & $6.66 *$ & $6.30 *$ & 7.02 & 5.76 & 7.56 & 79.0 & 73.0 & 98.5 & 107.0 \\
\hline $\mathrm{COAH}-026$ & I & $\mathrm{R}$ & $6.61 *$ & 5.61 & 5.98 & 5.68 & $9.17 *$ & 79.0 & 70.0 & 102.0 & 97.5 \\
\hline $\mathrm{COAH}-030$ & I & $\mathrm{R}$ & $6.59 *$ & 4.81 & 6.43 & $7.63 *$ & 7.50 & 78.0 & 72.0 & 94.0 & 103.0 \\
\hline \multicolumn{12}{|l|}{ Precoces } \\
\hline $\mathrm{COAH}-017$ & $\mathrm{~T}$ & $\mathrm{CN}$ & 5.20 & 2.86 & 4.43 & 6.45 & 7.07 & 67.0 & 67.0 & 78.0 & 88.0 \\
\hline $\mathrm{COAH}-050$ & I & $\mathrm{R}$ & 5.18 & 4.01 & 5.78 & 4.63 & 6.28 & 72.0 & 59.5 & 87.0 & 91.5 \\
\hline COAH-012 & A & $\mathrm{CN}$ & 4.39 & 2.65 & 4.69 & 5.01 & 5.21 & 66.0 & 61.0 & 79.0 & 81.0 \\
\hline COAH-052 & I & $\mathrm{R}$ & 4.25 & 3.64 & 3.75 & 4.06 & 5.57 & 72.5 & 59.5 & 80.0 & 89.5 \\
\hline COAH-054 & I & $\mathrm{R}$ & 3.89 & 2.73 & 3.90 & 3.24 & 5.67 & 67.5 & 58.0 & 88.0 & 87.0 \\
\hline Media & & & 5.76 & 4.77 & 5.44 & 5.71 & 7.13 & 77.8 & 71.0 & 96.6 & 102.0 \\
\hline $\mathbf{E E}$ & & & 0.41 & 0.68 & 0.88 & 0.75 & 0.93 & 1.7 & 1.5 & 4.0 & 2.9 \\
\hline Máximo & & & 8.02 & 9.75 & 10.95 & 10.43 & 13.43 & 92.0 & 88.0 & 145.0 & 134.0 \\
\hline Mínimo & & & 2.59 & 0.59 & 1.13 & 2.56 & 2.71 & 65.0 & 57.0 & 78.0 & 80.0 \\
\hline
\end{tabular}

GC08 y GC09 = General Cepeda 2008 y 2009; MZ08 y MZ09 = Mezquite 2008 y 2009; Raza: C= Celaya; CN=Cónico Norteño; O = Olotillo; R = Ratón; T $=$ Tuxpeño; TN $=$ Tuxpeño Norteño; Grupo: $\mathrm{B}=$ Bajo; $\mathrm{I}=$ Intermedio; $\mathrm{T}=$ Transición; A=Altura; $*=$ Valores superiores a la $\mu+2$ el error estándar; $\mathrm{EE}=$ Error estándar. 
De esta manera se puede identificar a tres grupos de acuerdo con su adaptación: el primero, con adaptación a El Mezquite (valores positivos en la ordenada) con 30 poblaciones (33.4 \%); el segundo a la localidad de General Cepeda (valores negativos en la ordenada), con 38 poblaciones $(42.2 \%)$; y un tercer grupo de 22 poblaciones $(24.4 \%)$, con estabilidad a través de las dos localidades y dos años evaluados. La interacción poblaciones $\mathrm{x}$ ambientes (Figura 2) indica que el comportamiento promedio de los grupos raciales resultó asociado con los ambientes de evaluación. En El Mezquite, las razas con un comportamiento aceptable fueron Cónico Norteño (20.0 \%), Ratón (6.7 \%) y Tuxpeño Norteño (5.6 \%); en General Cepeda, las razas Ratón (17.8\%), Tuxpeño Norteño (12.2\%) y Tuxpeño (8.9\%); en el grupo de poblaciones estables, sobresale la raza Ratón $(12.2 \%)$, Cónico Norteño $(4.4 \%)$ y Tuxpeño Norteño $(4.4 \%)$.

Con base en el rendimiento promedio a través de ambientes, se identificó a las mejores poblaciones (Cuadro 3). Estas poblaciones son las que se encuentran con valores positivos en el eje de las abscisas (Figura 2), y corresponden principalmente a la raza Tuxpeño Norteño, la combinación de Tuxpeño Norteño con Ratón y Celaya; cuatro poblaciones de la raza Ratón y una de Tuxpeño; las combinaciones Ratón x Olotillo, Tuxpeño Norteño y Tuxpeño x Celaya. Entre los materiales estables y con buen potencial de rendimiento sobresalen las poblaciones 37 (Tuxpeño x Celaya), 66, 81, y 35 de la raza Tuxpeño Norteño y las poblaciones 38 y 64 (Ratón x Tuxpeño Norteño). De la misma manera, las poblaciones 19, 22, 34 y 61 tuvieron adaptación a la localidad El Mezquite, y en sentido opuesto las poblaciones 74, 76 y 88 con adaptación a General Cepeda.

La madurez de las poblaciones identificadas en el Cuadro 3 se ubica principalmente alrededor de la media poblacional, excepto las poblaciones 76,81 y 86 que son las más tardías. El análisis de los días a floración masculina permitió identificar a cinco poblaciones precoces y con buen potencial de rendimiento (Cuadro 3 ). Estas poblaciones son de la raza Ratón $(50,52$ y 54) y Cónico Norteño (12 y 17), y pueden ser utilizadas como fuente de precocidad en el mejoramiento genético.

\section{CONCLUSIONES}

Las poblaciones criollas de maíz 19, 22, 34 y 61 mostraron adaptación a El Mezquite; las poblaciones 74, 76 y 88 a General Cepeda; y las poblaciones 35, 37, 38, 64, 66 y 81 mostraron estabilidad a través de localidades y años. Los grupos raciales con mayor potencial de rendimiento fueron Tuxpeño, Tuxpeño Norteño y Ratón.

\section{AGRADECIMIENTOS}

A la Secretaría de Agricultura, Ganadería, Desarrollo Rural, Pesca y Alimentación (SAGARPA) y al Servicio Nacional de Inspección y Certificación de Semillas (SNICS), por el financiamiento del proyecto de investigación a través del Sistema Nacional de Recursos Fitogenéticos para la Alimentación y la Agricultura (SINAREFI)

\section{BIBLIOGRAFÍA}

Aguirre G J A, M R Bellon, M Smale (1998) A Regional Analysis of Maize Biological Diversity in Southeastern Guanajuato, Mexico. CIMMYT Economics Working Paper 98-06. CIMMYT, México, D.F. 17 p.

Barreto H J, G O Edmeades, S C Chapman, J Crossa (1997) The alpha lattice design in plant breeding and agronomy: generation and analysis. In: Proc. Symp. on Developing Drought- and Low N-Tolerant Maize. G O Edmeades, M Bänziger, H R Mickelson, C B Peña-Valdivia (eds). El Batan, Mexico, March 25- 29, 1996. CIMMYT. Mexico, D. F. pp:544-551.

Castillo F (1993) La variabilidad genética y el mejoramiento de los cultivos. Ciencia (México). pp:69-79.

García Z J, J Molina G, J López R (2002) La selección masal como método para obtener líneas de alta aptitud combinatoria específica en maíz. Rev. Fitotec. Mex. 25:299-304.

Hernández C J M, G Esquivel E (2004) Rendimiento de grano y características agronómicas en germoplasma de maíz de valles altos de México. Rev. Fitotec. Mex. 27 (Núm. Esp. 1):27-31.

IRRI (2007) CropStat for Windows 7.2.2007.3 1998-2007. International Rice Research Institute. Metro Manila, Philippines.

Márquez S F (2005) Consideraciones generales sobre el mejoramiento de maíces criollos. In: Mem. Primera Reunión de Mejoradores de Variedades Criollas de Maíz en México. L F Márquez O (ed). Exhacienda Nazareno, Xoxocotlán, Oax. 22-23 Sep. 2005. Centro Regional Universitario, Universidad Autónoma Chapingo. SOMEFI. Chapingo, Edo. de México. pp:153-162.

Ortega P R A, J J Sánchez G, F Castillo G, J M Hernández C (1991) Estado actual de los estudios sobre maíces nativos de México. In: Avance en el Estudio de los Recursos Fitogenéticos de México. Ortega P R, G Palomino H, F Castillo G, V A González H, M Livera M (eds). SOMEFI. Chapingo, México. pp:161-185.

SAGARPA-SIAP (2007) Situación Actual y Perspectivas del Maíz en México 1996-2012. Talleres Gráficos del Servicio de Información Agroalimentaria y Pesquera, México, D. F. 131 p.

SAS Institute (2004) SAS/STAT® 9.1 User's Guide. Cary, NC: SAS Institute Inc. USA. $5121 \mathrm{p}$.

Yan W (2007) GGEbiplot Pattern Explorer. The Complete Biplot Analysis System. Version 6.3 Ontario, Canada.

Yan W, M S Kang (2003) GGE Biplot analysis. A Graphical Tool for Breeders, Geneticists, and Agronomists. CRC Press LLC, New York. $271 \mathrm{p}$.

Yan W, N A Tinker (2006) Biplot analysis of multi-environment trial data: principles and applications. Can. J. Plant Sci. 86:623-645.

Wellhausen E J, L M Roberts, E Hernández X, en colaboración con Mangelsdorf (1951) Razas de Maíz en México. Su Origen, Características y Distribución. Folleto Técnico 5. Oficina de Estudios Especiales. Secretaría de Agricultura y Ganadería. México, D. F. 223 p. 